\title{
THE PROBLEM OF RADIOACTIVE PARTICLE BEAMS TRANSPORTATION AND THE EXPERIMENTAL REALIZATION OF THE PHENOMENON OF SUPPRESSED GAMMA-DECAY OF RADIOACTIVE NUCLEI
}

\author{
Vladimir I. Vysotskii \\ Kiev Shevchenko University, Radiophysical Faculty \\ Vladimirskaya St. 64, Kiev, Ukraine, 252033; e-mail viv@ vhome.kiev.ua
Vladimir P. Bugrov, Alla A. Kornilova, Sergei I. Reiman
Moscow State University, 119899, Moscow, Russia

\section{INTRODUCTION AND THEORETICAL MODEL}

The problem of acceleration and long-distance transportation of high-radioactive beams and short-lifetime radioactive nucleus beams is interesting for future beam and nuclear technologies. The method of short-lifetime radioactive nuclei and high-radioactive beams spontaneous decay braking and suppression at long distance transportation and acceleration is the most optimal solution of this problem. The paper discusses the successful experiments on controlling the probability of spontaneous gamma-decay and life-time of radioactive and excited nuclei of several isotopes..

It is well known that in free space (without adjacent material bodies) the radiative life-time $\tau$ of an excited nucleus is constant and equals

$$
\tau=3 \hbar / \mathrm{c}^{3} / 4 \omega_{\alpha}^{3}\left|\mathrm{~d}_{\mathrm{eg}}\left(\omega_{\alpha}\right)\right|^{2}
$$

The total life-time of this excited nucleus $\tau_{\text {tot }}=\tau /(1+\alpha)$ in free space is constant too. Here $d_{e g}$ is the matrix element of nucleus dipole momentum, $\alpha$ is the coefficient of internal electron conversion for nuclear transition $E_{e} \rightarrow E_{g}=0$ with frequency $\omega_{\alpha}=\left(E_{e}-E_{g}\right) / \hbar$.

We have created the theory of resonant screen influence on the gamma-decay probability and life-time of excited and radioactive nuclei ${ }^{1,2,3}$. The phenomenon of nuclear decay controlling is a result of interaction of the nucleus with zero-energy electromagnetic modes, which in turn interact with the screen. It was shown that the result of action of resonant $\left(\omega_{n 0} \approx \omega_{\alpha}\right)$ screen on the excited nucleus is the change of radiative and total life-times

$$
\begin{aligned}
& \tau \rightarrow \tau^{*} \equiv \tau / \operatorname{Re}\left[\left(1-2 \mathrm{i} \tau \Delta \omega_{0}\right) /\left(1+\mathrm{iG} / 2-\Delta \Omega_{\mathrm{r}}\right)^{4}\right] \\
& \tau_{\mathrm{tot}} \rightarrow \tau_{\text {tot }}^{*} \equiv \tau_{\mathrm{tot}}(\alpha+1) /\left\{\alpha+\operatorname{Re}\left[\left(1-2 \mathrm{i} \tau \Delta \omega_{0}\right) /(1+\mathrm{iG} / 2-\right.\right. \\
& \left.\left.\left.\Delta \Omega_{\mathrm{r}}\right)^{4}\right]\right\}= \\
& \tau /\left\{\alpha+\operatorname{Re}\left[\left(1-2 \mathrm{i} \tau \Delta \omega_{0}\right) /\left(1+\mathrm{iG} / 2-\Delta \Omega_{\mathrm{r}}\right)^{4}\right]\right\}, \\
& \text { Here } \\
& \mathrm{G} \approx 2 \mathrm{fN} \pi^{2}\left|\mathbf{d}_{\mathrm{eg}}\left(\omega_{\mathrm{a}}\right)\right|^{2} / 3 \tau_{\text {tot }}^{*} \hbar \mathrm{v}_{0}\left[\left(\omega_{\mathrm{n} 0}-\omega_{\mathrm{a}}\right)^{2}+\left(1 / 2 \tau_{\mathrm{tot}}^{*}\right)^{2}\right], \\
& \Delta \Omega_{\mathrm{r}} \approx 2 \mathrm{fN} \pi\left|\mathbf{D}_{\mathrm{eg}}\left(\omega_{\mathrm{n} 0}\right)\right|^{2}\left(\omega_{\mathrm{n} 0}-\omega_{\mathrm{a}}\right) / 3 \hbar \mathrm{v}_{0}\left[\left(\omega_{\mathrm{n} 0}-\omega_{\mathrm{a}}\right)^{2}+\right. \\
& \left.\qquad\left(1 / 2 \tau_{\mathrm{tot}}\right)^{2}\right] \equiv\left(\omega_{\mathrm{n} 0}-\omega_{\mathrm{a}}\right) \mathrm{G} \tau_{\mathrm{tot}}^{*} / \pi,
\end{aligned}
$$

$\mathbf{D}_{\mathrm{eg}}$ is a matrix element of screen atom dipole momentum, $v_{0}$ is a volume of electromagnetic mode quantization, $\mathrm{f}$ is the Mossbauer parameter,

$$
\Delta \omega_{0}=\hat{P} \int_{0}^{\infty} 2 \omega_{\alpha}{ }^{3}\left|\mathbf{d}_{\mathrm{eg}}\left(\omega_{\alpha}\right)\right|^{2} \mathrm{~d} \omega_{\alpha} / 3 \pi c^{3}\left(\omega_{\alpha}-\omega_{\mathrm{a}}\right)
$$

— radiative shift of excited level energy of the nucleus,

$$
\begin{gathered}
\mathrm{G}=2 \mathrm{~N} \pi^{2}\left|\mathbf{d}_{\text {ege }}\left(\omega_{\mathrm{n} 0}\right)\right|^{2} / 3 \tau_{\text {atot }} \hbar \mathrm{v}_{0}\left[\left(\omega_{\mathrm{n} 0}-\omega_{\mathrm{a}}\right)^{2}+\left(1 / 2 \tau_{\text {atot }}\right)^{2}\right] \\
\Delta \Omega_{\mathrm{r}}=\left(\omega_{\mathrm{ns}}-\omega_{\mathrm{a}}\right) \mathrm{G} \tau_{\text {atot }} / \pi .
\end{gathered}
$$

For $|\mathrm{G}|,\left|\Delta \Omega_{\mathrm{r}}\right|<<1$ we have $\tau_{\text {tot }}^{*}=\tau /\left[\alpha+1-8 \mathrm{f} \tau \Delta \omega_{0} \mathrm{~N} \pi^{2} \mathrm{c}^{3} /(\alpha+1) \mathrm{v}_{0} \omega_{\mathrm{n} 0}{ }^{3}\right]=$

$\tau /\left[\left(\alpha+1-\left(1-\tau / \tau^{*}\right) \mathrm{f} \Delta \Theta / 4 \pi\right)\right]$,

$\tau^{*}=\tau /\left[1-\mathrm{f} \tau \Delta \omega_{0} \mathrm{~N} \pi^{2} \mathrm{c}^{3} /(\alpha+1) \mathrm{v}_{0} \omega_{\mathrm{n} 0}{ }^{3}\right]$.

For the case of nonresonant $\left(\omega_{\mathrm{ns}} \neq \omega_{\alpha}\right)$ screen

$\tau_{\text {tot }}^{*}=\tau /\left[\alpha+\left(1-\Delta \Omega_{\mathrm{nr}}\right)^{-4}\right]$;

$$
\begin{aligned}
& \quad \Delta \Omega_{\mathrm{nr}}=2 \pi \sum_{\mathrm{s}} \mathrm{N}_{\mathrm{s}}\left|\mathbf{D}_{\mathrm{eg}}\left(\omega_{\mathrm{ns}}\right)\right|^{2}\left(\omega_{\mathrm{ns}}-\omega_{\mathrm{a}}\right) / 3 \hbar \mathrm{v}_{0 \mathrm{~s}}\left[\left(\omega_{\mathrm{ns}}-\omega_{\mathrm{a}}\right)^{2}\right. \\
& \left.+\left(2 \tau_{\text {tot }}^{*}\right)^{-2}\right]
\end{aligned}
$$

It was shown that resonant screen effect in all cases appears to be more significant than for the nonresonant one. For the same relation $\mathrm{N} / \mathrm{V}_{0}$ the influence of the resonant screen upon life-times $\tau_{\text {tot }}^{*}, \tau^{*}$ of excited nucleus is by several orders more effective than the influence of the nonresonant screen.

Two experiments on controlling of the nuclei decay and changing $\tau_{\text {tot }}^{*}, \tau^{*}$ were performed based on our theory.

\section{THE EXPERIMENTAL STUDY OF THE PHENOMENON OF CONTROLLING TIME-LIFE OF SPONTANEOUS DECAY OF EXCITED NUCLEI BY MOSSBAUER SPECTRUM INVESTIGATION}

The aim of the first experiment was to measure the changing (decreasing at $\omega_{n 0}=\omega_{\mathrm{a}}$ ) of the spectral width of Mossbauer radiation $\Gamma \equiv 1 / \tau_{\text {tot }} \rightarrow \Gamma^{*} \equiv 1 / \tau_{\text {tot }}^{*}$, (as a result of 
changing life-times $\tau_{\text {tot }}^{*}$ and $\tau^{*}$ ) during action of resonant screen. The layouts of the experiments are presented on Fig.1.

The anticipated change in radiative and total widths of gamma-spectrum increases with the decrease of life-time $\tau$. In order to reduce the influence of technical fluctuations the Mossbauer isotope $\mathrm{Sn}^{119 \mathrm{~m}}$ with short total $\tau_{\text {tot }}=1,85.10^{-8} \mathrm{~s}$ and radiative $\tau=1,2.10^{-7} \mathrm{~s}$ life-times was used. The experimental installation was mounted on the double layer of acoustic isolation.

The excited $\mathrm{Sn}^{119 \mathrm{~m}}$ isotope (chemical compound $\mathrm{CaSn}{ }^{119 \mathrm{~m}} \mathrm{O}_{3}$ ) with activity of $5 \mathrm{mCi}$ and $\alpha=5,5$ was used as a source 1 of Mossbauer radiation with the energy of quanta $\mathrm{E}_{\gamma}=23,8 \mathrm{KeV}$. This source had a radiation spectrum in the form of a single line of nearly natural width.

The resonant absorber 2 had a form of disk with diameter $\mathrm{D} \approx 3 \mathrm{~cm}$, made of stable $\mathrm{Sn}^{119}$ isotope (average surface density $\sigma_{\mathrm{m}} \approx 1,4 \mathrm{mg} / \mathrm{cm}^{2} \approx 6.10^{18}$ atoms $\mathrm{Sn}^{119}$ per $\mathrm{cm}^{2}$, chemical compound $\mathrm{Sn}^{119} \mathrm{O}_{2}$ ). This absorber (screen) had a spectrum of absorption with almost natural width.

The lead diaphragm 3 with diameter $\mathrm{D}_{1} \approx \mathrm{D}$ was fixed at distance $\mathrm{L}=3 \mathrm{~cm}$ from source. The diaphragm had a hole with diameter $\mathrm{D}_{0}=1 \mathrm{~cm}$ and length $\mathrm{L}_{0}=2,5 \mathrm{~cm}$. Behind the diaphragm there was a resonant detector 4 (a compound $\mathrm{CaSn}^{119} \mathrm{O}_{3}$ was used as this detector) and a system for changing the Doppler velocity of detector 4.

The measurements with gamma-beam (traveling from source through resonant absorber and hole of the diaphragm to resonant detector) were performed in two regimes.

In the first regime the resonant absorber 2 was fixed in position a) near source 1 (at distance $l_{1} \approx 0,2 \mathrm{~cm}$ ). The finite quantizing volume $\mathrm{V}_{0}$ in this regime existed only for those electromagnetic modes which were localized in solid angle between source and the diaphragm. The volume of each of these modes corresponded to the value $\mathrm{V}_{0} \approx \pi \lambda \mathrm{L}^{2} / 3$ of space between two cones with common apex on the excited nucleus that cut a single Fresnel zone with area $S_{0} \approx \pi \lambda L$ on the diaphragm surface. Only for
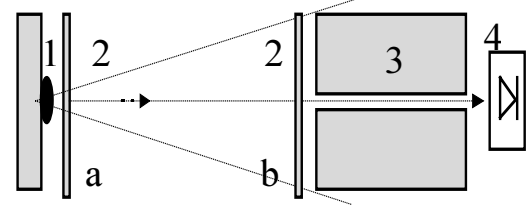

Fig 1

these modes $\tau_{\text {tot }}^{*} \neq \tau_{\text {tot }}, \tau^{*} \neq \tau$.

For other space directions and for other space mode orientations we have $\mathrm{V}_{0} \rightarrow \infty$. In accordance with (4) and (5) in these cases of modes unlimited in space we have $\tau_{\text {tot }}^{*}=\tau_{\text {tot }}, \tau^{*}=\tau$.

In the second regime the resonant absorber 2 was fixed at distance $l_{2} \approx 3 \mathrm{~cm}$ from source in position b) near the diaphragm $3\left(l_{2} \approx \mathrm{L}\right)$.

The relation $\mathrm{N} / \mathrm{V}_{0}$ was different in these two regimes.

For the first regime
$\left(\mathrm{N} / \mathrm{V}_{0}\right)_{\mathrm{a}} \approx 3 \sigma_{\mathrm{m}} l_{1}^{2} / \mathrm{L}^{3} \approx 2,7.10^{16} \mathrm{~cm}^{-3}$

and

$\Delta \Theta_{\mathrm{a}} \approx 2 \pi\left\{1-1 /\left[1+\left(\mathrm{D}_{1} / 2 \mathrm{~L}\right)^{2}\right]^{1 / 2}\right\} \approx 0,4 \pi$.

For the second regime

$\left(\mathrm{N} / \mathrm{V}_{0}\right)_{\mathrm{b}} \approx 3 \sigma_{\mathrm{m}} / l_{2} \approx 6.10^{18} \mathrm{~cm}^{-3}$

and

$\Delta \Theta_{\mathrm{b}} \approx 2 \pi\left\{1-1 /\left[1+(\mathrm{D} / 2 \mathrm{~L})^{2}\right]^{1 / 2}\right\} \approx \Delta \Theta_{\mathrm{a}} \approx 0,4 \pi$.

In the regimes (a) and (b) the total widths $\Gamma_{(\mathrm{a}, \mathrm{b})}^{*}=\Gamma_{\mathrm{s}(\mathrm{a}, \mathrm{b})}^{*}+\Gamma_{\mathrm{d}}+\Delta \Gamma$ of gamma-spectrums included both the sum of source $\left(\Gamma_{\mathrm{s}(\mathrm{a}, \mathrm{b})}^{*}\right)$ and resonant detector $\left(\Gamma_{\mathrm{d}}\right)$ resonant-line widths, and the broadening $\Delta \Gamma$ (as a result of resonant absorption) in absorber 2.

In order to verify the stability of gamma-line position and width the measurement of gamma-spectrum was performed in the system of source 1 - hole of diaphragm 3 - resonant detector 4 without the resonant absorber 2 . The resulting value

$\Gamma_{\mathrm{s}}+\Gamma_{\mathrm{d}}=\Gamma_{\mathrm{s} 0}+\Delta \Gamma_{\mathrm{so}}+\Gamma_{\mathrm{d} 0}+\Delta \Gamma_{\mathrm{do}} \approx(0.809 \pm 0.002) \mathrm{mm} / \mathrm{s}$ (in Doppler velocity units $\mathrm{c} \Gamma / \omega_{\mathrm{a}}$ ) for this case demonstrates that the nonradiative broadening $\Delta \Gamma_{\mathrm{so}} \approx \Delta \Gamma_{\mathrm{do}} \approx 0,081 \mathrm{~mm} / \mathrm{s}$ in the source and detector is small in relation to natural widths $\Gamma_{\mathrm{s} 0}=\Gamma_{\mathrm{do}}=1 / \tau_{\mathrm{tot}} \approx 0,323 \mathrm{~mm} / \mathrm{s}$. It follows from these results that there are no sources of technical nonstability and systematic errors in the experimental instalation.

For the regime (a) we have

$\Gamma_{\mathrm{s} 0} \rightarrow \Gamma_{\mathrm{s} 0(\mathrm{a})}^{*} \equiv 1 / \tau_{\text {tot(a) }}^{*}, \Gamma_{\mathrm{s}(\mathrm{a})}^{*} \rightarrow \Gamma_{\mathrm{s}(\mathrm{a})}^{*}+\Delta \Gamma_{\mathrm{so}}$.

As a result for this regime we have the following

expression for the total width of gamma-spectrum

$\Gamma^{*}{ }_{(\mathrm{a})}=1 / \tau_{\text {tot(a) }}^{*}+\Delta \Gamma_{\mathrm{so}}+\Gamma_{\mathrm{d} 0}+\Delta \Gamma_{\mathrm{do}}+\Delta \Gamma$.

For the regime (b) with presence of the resonant absorber 2 near the diaphragm we have the similar result for the total width of gamma-spectrum

$$
\Gamma_{(\mathrm{b})}^{*}=1 / \tau_{\mathrm{tot}(\mathrm{b})}^{*}+\Delta \Gamma_{\mathrm{so}}+\Gamma_{\mathrm{d} 0}+\Delta \Gamma_{\mathrm{do}}+\Delta \Gamma \text {. }
$$

Then (using the results of theoretical calculation (8), (9) for $\Gamma_{(\text {a) }}^{*}$ and $\Gamma_{(\text {b) }}^{*}$ and equations (3) - (5)) we find the expressions that describe the changing of total and radiative (for controlled Mossbauer part) life-times

$$
\begin{aligned}
& 1 / \tau_{\text {tot(b) }}^{*}-1 / \tau_{\text {tot(a) }}^{*}=\Gamma_{(\mathrm{b})}^{*}-\Gamma_{(\mathrm{a}),}^{*}, \\
& 1 / \tau_{\text {ef(b) }}^{*}-1 / \tau_{\text {ef(a) }}^{*}=\left(\Gamma_{(\mathrm{b})}^{*}-\Gamma_{(\mathrm{a}))}^{*}\right)[4 \pi(1+\alpha) / \mathrm{f} \Delta \Theta]
\end{aligned}
$$

At small change of life-time $\left|\tau_{\text {tot(a) }}^{*}-\tau_{\text {tot(b) }}^{*}\right|<<\tau_{\text {tot }}$ we have

$\left(\tau_{\text {tot(a) }}^{*}-\tau_{\text {tot(b) }}^{*}\right) / \tau_{\text {tot }} \approx\left(\Gamma_{(\mathrm{b})}^{*}-\Gamma_{(\mathrm{a})}^{*}\right) \tau_{\mathrm{tot}}$,

$\tau / \tau_{\text {ef(b) }}^{*}-\tau / \tau^{*}{ }_{\text {ef(a) }}=\left(\Gamma_{\text {(b) }}{ }^{*}-\Gamma_{(\mathrm{a})}{ }^{*}\right) \tau_{\text {tot }}[4 \pi(1+\alpha) / \mathrm{f} \Delta \Theta]$

Using the expression (4) and the results of measuring

$\tau_{\text {tot(a,b) }}{ }^{*}$ we can calculate the radiative shift

$$
\Delta \omega_{0} \approx\left\{\left(\tau_{\text {tot(a) }}^{*}-\tau_{\text {tot(b) })}^{*}\right) / \tau_{\text {tot }}\right\}(\alpha+1)^{2} \omega_{\mathrm{n} 0}{ }^{3} /\left\{2 \mathrm { f } \tau \pi \mathrm { c } ^ { 3 } \left[\Delta \Theta_{\mathrm{a}}\right.\right.
$$

$\left.\left.\left(\mathrm{N} / \mathrm{V}_{0}\right)_{\mathrm{a}}-\Delta \Theta_{\mathrm{b}}\left(\mathrm{N} / \mathrm{V}_{0}\right)_{\mathrm{b}}\right]\right\}$.

Each measurement of $\Gamma^{*}$ (a) and $\Gamma_{(\text {b) }}^{*}$ in both cases lasted $\Delta \mathrm{t}=0,5$ hour. The average values measured were

$\Gamma_{(\mathrm{a})}^{*}=(1,167 \pm 0,003) \mathrm{mm} / \mathrm{s}$ and

$\Gamma_{(\mathrm{b})}^{*}=(1,184 \pm 0,003) \mathrm{mm} / \mathrm{s}$ with corresponding changes of total life-time of $\mathrm{Sn}^{119 \mathrm{~m}}$

$\left\{\tau_{\text {tot(a) }}^{*} \tau_{\text {tot(b) })}^{*}\right\} / \tau_{\text {tot }}=(0,63 \pm 0,12) \cdot 10^{-2}$

and life-time for controlled Mossbauer component of $\mathrm{Sn}^{119 \mathrm{~m}}$ gamma-radiation 
$\left\{\tau / \tau_{\text {ef(b) })}^{*} \tau / \tau_{\text {ef(a) }}^{*}\right\}=(0,82 \pm 0,16)$.

Using the result of measuring $\left(\tau_{\text {tot(a) }}{ }^{*}-\tau_{\text {tot(b) }}{ }^{*}\right) / \tau_{\text {tot }}$ and the general expression (12) we for the first time have obtained the magnitude and sign of radiative shift (radiation correction) of excited nucleus $\mathrm{Sn}^{119 \mathrm{~m}}$ level

$\Delta \omega_{0} \approx-(2,9 \pm 0,55) \cdot 10^{14} \mathrm{~s}^{-1}$.

The measures taken to improve the reliability of measurements in the "frequency-response" regime allow to connect with a high degree of certainty the experimental results with theoretically predicted controlled charge of nucleus gamma-decay probability and life-time and not with false factors.

\section{THE EXPERIMENTAL STUDY OF THE PHENOMENON OF CONTROLLED SPONTANEOUS GAMMA-DECAY OF RADIOACTIVE NUCLEI BY INVESTIGATION OF INTENSITY OF GAMMA-RADIATION}

The aim of the second experiment was to measure the changing of total intensity $\mathrm{J}_{\gamma} \rightarrow \mathrm{J}_{\gamma}{ }^{*}$ of resonant nuclei radiation (as a result of changing life-times $\tau \rightarrow \tau^{*}$ ) in the direction $(4 \pi-\Delta \Omega)$ not blocked by the resonant screen 2 . Equation for population $\mathrm{n}_{\mathrm{e}}{ }^{*}$ of excited nuclei has a form

$$
\begin{gathered}
\mathrm{dn}_{\mathrm{e}}{ }^{*} / \mathrm{dt}=\Sigma_{\mathrm{i}>\mathrm{e}} \mathrm{n}_{\mathrm{i}} / \tau_{\mathrm{i} 2}-\mathrm{n}_{\mathrm{e}}{ }^{*} \Delta \Omega \mathrm{f} / 4 \pi \tau^{*}-\mathrm{n}_{\mathrm{e}}{ }^{*}(1-\mathrm{f}) / \tau- \\
\mathrm{n}_{\mathrm{e}} \mathrm{f}(1-\Delta \Omega / 4 \pi) / \tau-\mathrm{n}_{\mathrm{e}} \mathrm{f} \alpha / \tau .
\end{gathered}
$$

Here $\Delta \Omega$ is a solid angle blocked by the absorber.

In equilibrium state the relation of population of excited nuclei $\mathrm{n}_{2}{ }_{2}$ for $\Delta \Omega \neq 0$ (with selective absorption) to population of excited nuclei $\mathrm{n}_{2}$ for $\Delta \Omega=0$ (without selective absorption) has a form

$$
\mathrm{n}_{2}{ }^{*} / \mathrm{n}_{2}=1 /\left\{1-\mathrm{f} \Delta \Omega\left(1-\tau_{2} / \tau_{2}{ }^{*}\right) / 4 \pi(1+\alpha)\right\} .(14)
$$

Intensity $\mathrm{J}_{\gamma}{ }^{*} \sim \mathrm{n}_{\mathrm{t}}{ }^{*}$ of resonant radiation (Mossbauer's probability $\mathrm{f}$ ) in not blocked direction increase and

$$
\mathrm{J}_{\gamma}{ }^{*} / \mathrm{J}_{\gamma}=\mathrm{n}_{\mathrm{e}}{ }^{*} / \mathrm{n}_{\mathrm{e}}=1 /\left[1-\mathrm{f}\left(1-\tau / \tau^{*}\right) \Delta \Omega / 4 \pi(1+\alpha)\right] .(15)
$$

The layout of the experiment is presented on Fig.2.
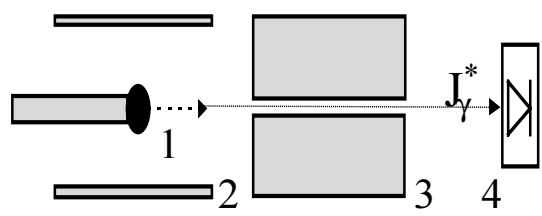

Fig 2
A excited $\mathrm{Co}^{57}\left(\mathrm{Fe}^{57 *}\right)$ isotope source 1 of spontaneous radiation $\left(\tau \approx 10^{-6} \mathrm{~s}, \tau_{\text {tot }}=\tau / \mathrm{W} \approx 10^{-7} \mathrm{~s}, \hbar \omega=14,4 \mathrm{KeV}\right)$ with activity of $10 \mathrm{mCi}$ in a chromium matrix was used as a source of Mossbauer radiation. This source has a spectrum in the form of single line of natural width.

The source was put in the center or near the diaphragmoriented edge of the resonant screen 2, having a form of cylinder made of stable $\mathrm{Fe}^{57}$ isotope. The source is fixed in the Plexiglas disc and put in the center $(l=2.5 \mathrm{~cm})$ or near the edge $(l=1 \mathrm{~cm})$ of the resonant absorber 2, having a form of cylinder with diameter $\mathrm{D}=2 \mathrm{~cm}$ and length $\mathrm{L}=5 \mathrm{~cm}$, made of Fe57 isotope $(200 \mathrm{mg})$ in stainless steel $(100 \mathrm{mg})$. The thickness of absorber was $7 \mathrm{mg} / \mathrm{cm}^{2}$. The lead diaphragm 3 has a hole with diameter $\mathrm{D}_{0}=1 \mathrm{~cm}$ and length $\mathrm{L}_{0}=2,5 \mathrm{~cm}$. Behind the diaphragm there was amplitude detector 4 - $\mathrm{NaJ}(\mathrm{Tl})$ crystal with width $10^{-2}$ $\mathrm{cm}$ and photo-electronic multiplier .

The signal processing system picked out the part of amplitude specter close to the gamma-line with $\mathrm{E}_{\gamma}=14,4$ $\mathrm{KeV}$.

For the first time in this experiment we have discovered the change of radiative transition life-time $\tau^{*}$ by $40-100 \%$ and total life-time $\tau_{\text {tot }}^{*}$ (including non-resonant radiation with probability 1-f and non-radiative channel of decay with probability $\mathrm{W}$ ) by $0.6-2 \%$.

\section{CONCLUSION}

By optimization of decay controlling system parameters (using the nuclei without the electron conversion decay channel $\alpha \rightarrow 0$, the resonance absorbers with a maximum solid angle screening $\Delta \Omega \rightarrow 4 \pi$, with maximum weight part of the gamma-radiative resonance channel $f \rightarrow 1$ ) it is possible to achieve significantly higher influence upon the spontaneous decay characteristics and, respectively, sharp increase of total life-time $\tau_{\text {tot }}{ }^{*}>>\tau_{\text {tot }}$.

Among other conclusions, the obtained results in fact prove the existence of a peculiar macroscopic "distance effect" predicted above, namely the dependence of the effectiveness of quantum spontaneous decay process of the excited nuclei on macroscopically remote position of a resonant absorbing screen (unlike, for example, Kazimir's effect manifesting itself only at microscopic distances).

In conclusion we would like to note that the effect of influence upon the spontaneous radiation characteristics of excited (radioactive) nuclei may manifest itself not only for Mossbauer nuclei and transitions but also for other excited states and nucleus types provided the existence for them of an obviously expressed resonance absorbtion. By this method of spontaneous decay braking and suppression of short-life-time radioactive nuclei and high-radioactive beams it is possible long distance transportation and acceleration of redioactive beam.

\section{REFERENCES}

1. V.I. Vysotskii, R.N.Kuzmin On change of characteristics of spontaneous radiation at reformation of electromagnetic vacuum, Soviet Phys - J.T.P. Letter, 10, 300 (1984)

2. V.I. Vysotskii, V.P. Bugrov, A.A.Kornilova, R.N.Kuzmin, S.I Reiman , "The problem of gamma-laser and controlling of Mossbauer nuclei decay (theory and practica)", Hyperfine Interactions, 107, 277 (1997)

3. V.I. Vysotskii, "The problem of controlled spontaneous nuclear gamma-decay: theory of controlled excited and radioactive nuclei gamma-decay,". Phys. Rev. C, 58, 337 (1998) 